\title{
Comparison of anti-anaerobic antimicrobial strategies in cancer patients with febrile neutropenia and gastrointestinal symptoms
}

Regis G Rosa ${ }^{1}$, Rodrigo P dos Santos ${ }^{2}$ and Luciano Z Goldani ${ }^{1,3^{*}}$

\begin{abstract}
Background: The current study sought to compare 28-day mortality rates in cancer patients with febrile neutropenia (FN) and gastrointestinal (GI) symptoms who underwent monotherapy using an antibiotic with antipseudomonal and anti-anaerobic activity (piperacillin-tazobactam or a carbapenem) and a group treated with a combination of cefepime-metronidazole.

Findings: We performed a prospective cohort study in a single tertiary hospital from October 2009 to August 2011. All consecutive adult cancer patients admitted with FN secondary to intensive chemotherapy and Gl symptoms (abdominal pain, diarrhea or perianal pain) were evaluated. Kaplan-Meier curves were used for calculating time-dependent occurence of death. In total, 37 patients with FN and Gl symptoms were evaluated (15 in monotherapy arm and 22 in the combination therapy arm). Treatment with combination cefepime and metronidazole resulted in a lower 28-day mortality rate compared with piperacillin-tazobactam or carbapenem monotherapy ( $0 \%$ versus $40 \%$; log-rank $P=0.002$ ).

Conclusions: Results of the present study suggest a significant reduction in mortality in cancer patients with FN and Gl symptoms treated with combination cefepime-metronidazole therapy compared with monotherapy using agents with antipseudomonal and anti-anaerobic activity. Further randomized trials are warranted to confirm the superior results using combination therapy in patients with FN and Gl symptoms.
\end{abstract}

Keywords: Bacteria, Anaerobic, Anti-bacterial agents, Febrile neutropenia, Immunocompromised host

\section{Findings}

Febrile neutropenia (FN) induced by cytotoxic chemotherapy is known to predispose patients to anaerobic bacteremia [1]. Damage to the gastrointestinal (GI) mucosa caused by anti-cancer agents provides a portal of entry for commensal anaerobic bacteria. This fact, in association with a decreased host response to infection due to granulocytopenia and impaired cellular immunity, may contribute to bloodstream infection caused by bacterial translocation [2]. Despite its low incidence, anaerobic bacteremia is usually associated with high morbidity and mortality rates, especially in the context of cancer and immunosuppression [3-5].

\footnotetext{
* Correspondence: Igoldani@ufrgs.br

${ }^{1}$ Graduate Program in Medicine: Medical Sciences, Universidade Federal do Rio Grande do Sul, Porto Alegre, Brazil

${ }^{3}$ Infectious Diseases Unit, Hospital de Clínicas de Porto Alegre, Ramiro

Barcelos 2350, Room: 700, Porto Alegre, RS 90640-000, Brazil

Full list of author information is available at the end of the article
}

Timely empirical therapy using an antipseudomonal, broad-spectrum antibiotic is part of the initial management of FN [6,7]. Anti-anaerobic coverage is usually recommended by guidelines as part of the initial treatment when GI symptoms (eg, abdominal pain, diarrhea, perianal pain) are present $[8,9]$. Unfortunately, only scarce data regarding the optimal anti-anaerobic antimicrobial strategy for patients with FN and GI symptoms are available. The aim of the present study was to compare the mortality rates of hospitalized adult cancer patients with FN and GI symptoms who either underwent monotherapy using an antibiotic with antipseudomonal and antianaerobic activity or were treated with a combination of cefepime and metronidazole.

\section{Study design and participants}

A prospective cohort study was conducted in a single tertiary centre from October 2009 to August 2011. All consecutive cancer patients admitted to the hematology 
ward of the Hospital de Clinicas de Porto Alegre (Porto Alegre, Brazil) with neutropenia (absolute neutrophil count $<500$ cells $/ \mathrm{mm}^{3}$, or $<1000$ cells $/ \mathrm{mm}^{3}$ with an expectation of a decrease to $<500$ cells $/ \mathrm{mm}^{3}$ during the ensuing $48 \mathrm{~h}$ ), fever (a single axillary temperature measurement $\geq 38.5^{\circ} \mathrm{C}$ or sustained temperature $\geq 38.0^{\circ} \mathrm{C}$ over a $1 \mathrm{~h}$ period) and GI symptoms (abdominal pain, presence of loose or watery stool, or perianal pain) were eligible for the present study. Subjects who were receiving only palliative treatment or who had an indication for outpatient treatment or neutropenia due to a specific etiology other than an adverse reaction to chemotherapy were excluded. Patients were not allowed to reenter the study after a first episode of FN with GI symptoms.

\section{Definitions}

The primary independent variable was the initial antimicrobial treatment administered by the medical care team. Due to observational study design, the research team did not influence treatment or diagnostic procedures. Patients were classified into two groups: subjects who received monotherapy using an antibiotic with antipseudomonal and anti-anaerobic activity (piperacillintazobactam $4.5 \mathrm{~g}$ intravenous [IV] over a $4 \mathrm{~h}$ period every $8 \mathrm{~h}$, or imipenem-cilastatin $500 \mathrm{mg}$ IV every $6 \mathrm{~h}$ or meropenem $1 \mathrm{~g} \mathrm{IV}$ every $8 \mathrm{~h}$ ); and subjects treated with a combination of cefepime 2 g IV every 8 h plus metronidazole $500 \mathrm{mg}$ IV every $8 \mathrm{~h}$. Clinical comorbidity was defined as the presence of heart failure, diabetes mellitus, chronic pulmonary disease or chronic liver disease. Nosocomial-acquired FN was defined as the onset of FN after 48 hours of hospitalization. High-dose chemotherapy was defined as induction chemotherapy or hematopoietic stem cell transplantation. The Multinational Association for Supportive Care in Cancer risk index score [10] was applied at the onset of fever to determine the risk for serious complications during an episode of FN; episodes were classified as high risk if the score was $<21$ and low risk if the score was $\geq 21$. Microbiological studies were performed at the onset of fever according to standards of practice and included two separate blood samples from two different anatomical sites for aerobic culture, and enzyme immunoassay testing for Clostridium difficile toxin A and B in a stool sample from patients with diarrhea. Antibiotic susceptibilities of the isolated pathogens were evaluated according to the recommendations of the Clinical and Laboratory Standards Institute [11]. Pseudomembranous colitis was diagnosed through a positive test for $C$. difficile toxin in a stool sample. Neutropenic enterocolitis was defined as bowel wall thickening $>4 \mathrm{~mm}$ at the terminal ileus, cecum or ascending colon documented by abdominal computed tomography performed in patients who experienced abdominal pain.

\section{Outcome and follow-up}

The primary outcome measure of the present study was mortality 28 days after the onset of FN. Patients were followed-up through interviews and medical record reviews using a standardized data collection instrument by researchers who were not associated with the assistant physician's team. Follow-up was maintained for 28 days after the onset of fever in neutropenic patients. For subjects who were discharged before 28 days, follow-up telephone calls were made on the 28th day after the onset of FN to determine whether they remained alive.

\section{Statistical analysis}

The $X^{2}$ and Fisher's exact tests were used to compare categorical variables; the Mann-Whitney $U$ test was used to compare continuous variables. Kaplan-Meier curves were used to calculate the time-dependent occurrence of death. The log-rank test was used for comparisons between groups. Statistical analysis was performed using STATA version 12 (Stata Corp LP, USA).

\section{Ethics issues}

The Institutional Review Board of the Hospital de Clínicas de Porto Alegre approved the study and written informed consent was obtained from all study participants.

\section{Results}

In total, 37 patients were evaluated during the study period; patients with hematological malignancies comprised $75 \%$ of the study population. The predominant neoplastic diseases were acute myeloid leukemia (35.1\%), lymphoma (21.6\%), acute lymphoblastic leukemia (16.2\%) and multiple myeloma (16.2\%). The proportion of patients who underwent high-dose chemotherapy was $56.7 \%$. Antibiotic prophylaxis with fluoroquinolones was not administered to any patient. Fifteen subjects initially underwent monotherapy using an antibiotic with antipseudomonal and anti-anaerobic activity (12 received piperacillintazobactam; three received a carbapenem), while 22 subjects were initially treated with a combination of cefepime and metronidazole. The overall mortality rate in the present cohort was $16.2 \%$ (six patients).

Characteristics of the patients who underwent monotherapy using an antibiotic with antipseudomonal and anti-anaerobic activity and those treated with a combination of cefepime and metronidazole are presented in Table 1 . There were no statistically significant differences between the two groups with regard to baseline characteristics. The incidence of neutropenic enterocolitis, pseudomembranous colitis, aerobic bacteremia and in vitro resistance of aerobic blood isolates to initial antibiotic treatment was also similar for the two study arms.

The 28-day mortality rate was significantly lower in the combination therapy group compared with the 
Table 1 Characteristics of patients with febrile neutropenia (FN) and gastrointestinal symptoms

\begin{tabular}{|c|c|c|c|}
\hline Variable & $\begin{array}{l}\text { Combination therapy }{ }^{a} \text { group } \\
(n=22)\end{array}$ & $\begin{array}{c}\text { Monotherapy }{ }^{b} \text { group } \\
(n=15)\end{array}$ & $P$ \\
\hline Age, years, mean (SD) & $44.7(14.3)$ & $41.2(11.0)$ & 0.43 \\
\hline Female sex & $15(68.1)$ & $5(33.3)$ & 0.05 \\
\hline Type of Cancer & & & $0.18^{c}$ \\
\hline Acute myeloid leukemia & $6(27.3)$ & $7(46.7)$ & \\
\hline Acute lymphoblastic leukemia & $4(18.2)$ & $2(13.3)$ & \\
\hline Chronic myeloid leukemia & $1(4.5)$ & $2(13.3)$ & \\
\hline Multiple myeloma & $6(27.3)$ & $0(0)$ & \\
\hline Lymphoma & $5(22.7)$ & $3(20.0)$ & \\
\hline Other solid tumors & $0(0)$ & $1(6.7)$ & \\
\hline Relapsing underlying disease & $13(59.0)$ & $12(80.0)$ & 0.28 \\
\hline Clinical comorbidity & $5(22.7)$ & $7(46.6)$ & 0.16 \\
\hline High dose chemotherapy regimens & $12(54.5)$ & $9(60.0)$ & 0.74 \\
\hline Nosocomial-acquired episode of FN & $19(86.3)$ & $13(86.6)$ & 0.97 \\
\hline ANC at the time of diagnosis of $\mathrm{FN}$, median cells $/ \mathrm{mm}^{3}$ (IQR) & $80(160)$ & $130(360)$ & 0.47 \\
\hline ANC $<100$ cells $/ \mathrm{mm}^{3}$ at the time of diagnosis of FN & $13(59.0)$ & $6(40.0)$ & 0.32 \\
\hline High-risk MASCC score & $8(36.3)$ & $2(13.3)$ & 0.15 \\
\hline Neutropenic enterocolitis & $1(4.5)$ & $1(6.6)$ & 0.99 \\
\hline Pseudomembranous colitis & $1(4.5)$ & $1(6.6)$ & 0.99 \\
\hline Documented aerobic bacteremia & $8(36.3)$ & $9(60.0)$ & 0.19 \\
\hline In vitro resistance of aerobic blood isolates to initial antibiotic treatment & $1(4.5)$ & $2(13.3)$ & 0.55 \\
\hline
\end{tabular}

monotherapy group ( $0 \%$ versus $40.0 \%$; log rank $P=0.002)$ (Figure 1). The assessment of whether mortality was attributable to infection was concordant in all six patients who died.

\section{Conclusions}

The present study demonstrated higher survival rates in adult cancer patients with FN who presented with GI symptoms treated with a combination of a fourth-generation cephalosporin plus metronidazole compared with those who underwent monotherapy using a broad-spectrum antibiotic with antipseudomonal and anti-anaerobic activity.

The findings of this study have scientific plausibility given that an antimicrobial strategy using a combination of cefepime and metronidazole has better coverage for anaerobic pathogens than a monotherapy strategy (piperacillin-tazobactan or a carbapenem). This hypothesis is supported by the fact that, in our study, the incidence of aerobic bacteremia and the proportion of in vitro resistance of aerobic blood isolates to initial antibiotic treatment were similar in the two treatment arms. Moreover, previous efficacy studies involving distinct populations have reported results convergent with those obtained in the present cohort. In a randomized clinical trial involving 122 patients with intra-abdominal infections, Garbino et al. [12] reported higher success rates with combination cefepime-metronidazole treatment compared with imipenem-cilastatin monotherapy $(87 \%$ versus $72 \% ; P=0.004)$. Similarly, Barie et al. [13] reported

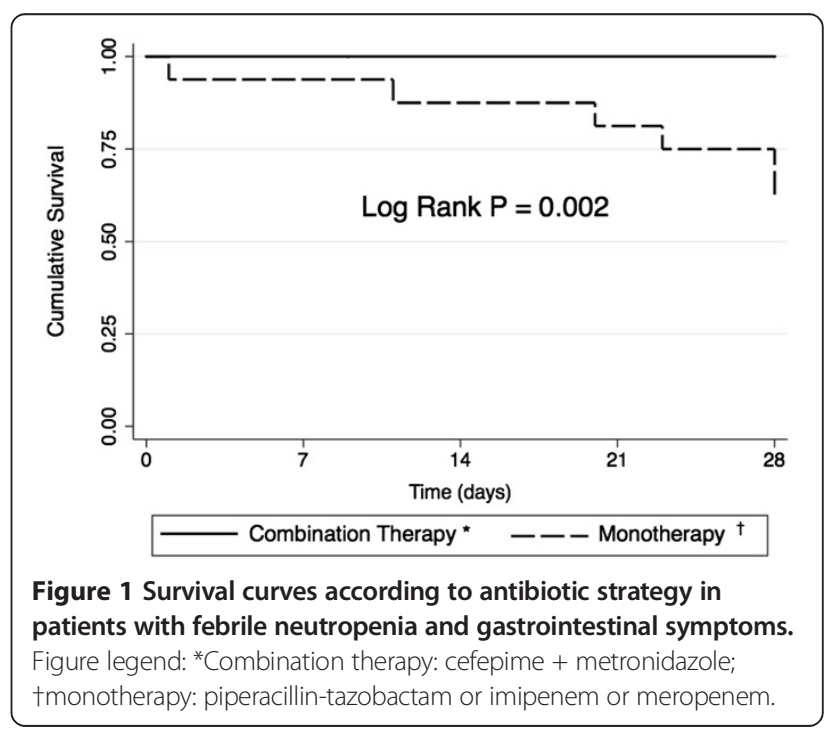


higher cure rates for combination treatment with cefepime plus metronidazole compared with imipenemcilastatin treatment in adult subjects with complicated intra-abdominal infections (88\% versus $76 \% ; P=0.02$ ). The high impact in risk reduction of mortality found in our study using combination cefepime-metronidazole treatment may be due the high levels of characteristically expected morbidity and mortality of our study population, which consisted of a large proportion of high-risk neutropenic patients in whom the correct choice of antimicrobial strategy is of paramount importance. In addition, considering the low incidence of $C$. difficile colitis in both arms of our study, empirical addition of metronidazole to the initial regimen suggests improved efficacy against anaerobes other than $C$. difficile in this setting. In fact, metronidazole has good activity against pathogenic anaerobic bacteria, and a favourable pharmacokinetic and pharmacodynamic profile [14]; furthermore, combining metronidazole with cefepime in patients with FN and GI symptoms may provide additional advantages over monotherapy strategies using piperacillin-tazobactam or carbapenems because this combination is less expensive and has a lower potential of inducing antimicrobial resistance, especially in Gram-negative bacteria.

This study has some limitations, mainly related to the small study population and to the unicentre observational design; however, proper measurement of variables and outcomes with previously defined objective criteria, use of standardized data collection, as well as follow-up for research team that was not related to care, minimized the possibility of systematic errors.

Given the scarce data in the literature regarding this important issue, larger randomized trials are required to confirm the superiority of combination cefepimemetronidazole treatment over broad-spectrum monotherapies in patients with FN and GI symptoms.

\section{Abbreviations}

FN: Febrile Neutropaenia; Gl: Gastrointestinal; ANC: Absolute Neutrophill Count; MASCC: The Multinational Association for Supportive Care in Cancer.

\section{Competing interests}

The authors declare that they have no competing interests.

\section{Authors' contributions}

RGR, RPS and LZG are responsible for the concept, design and implementation of this study. RPS and LZG designed the methods of data collection. RGR coordinated data collection and performed data analysis. All authors read and approved the final manuscript.

\section{Acknowledgements}

The authors thank the data collection team and all patients who agreed to participate in this trial.

\section{Author details}

${ }^{1}$ Graduate Program in Medicine: Medical Sciences, Universidade Federal do Rio Grande do Sul, Porto Alegre, Brazil. IInfection Control Committee of Hospital de Clínicas, Universidade Federal do Rio Grande do Sul, Porto Alegre, Brazil. ${ }^{3}$ Infectious Diseases Unit, Hospital de Clínicas de Porto Alegre, Ramiro Barcelos 2350, Room: 700, Porto Alegre, RS 90640-000, Brazil.
Received: 15 August 2014 Accepted: 4 September 2014

Published: 8 September 2014

\section{References}

1. Lark RL, McNeil SA, VanderHyde K, Noorani Z, Uberti J, Chenoweth C: Risk factors for anaerobic bloodstream infections in bone marrow transplant recipients. Clin Infect Dis 2001, 33(3):338-343.

2. Taur $Y$, Pamer EG: The intestinal microbiota and susceptibility to infection in immunocompromised patients. Curr Opin Infect Dis 2013, 26(4):332-337. doi:10.1097/QCO.0b013e3283630dd3.

3. Ngo JT, Parkins MD, Gregson DB, Pitout JD, Ross T, Church DL, Laupland KB: Population-based assessment of the incidence, risk factors, and outcomes of anaerobic bloodstream infections. Infection 2013, 41(1):41-48 doi:10.1007/s15010-012-0389-4.

4. Cheng CW, Lin HS, Ye JJ, Yang CC, Chiang PC, Wu TS, Lee MH: Clinical significance of and outcomes for bacterioides fragilis bacteremia. J Microbiol Immunol Infect 2009, 42(3):243-250.

5. Papaparaskevas J, Katsandri A, Pantazatou A, Stefanou I, Avlamis A, Legakis NJ, Tsakris A: Epidemiological characteristics of infections caused by Bacterioides, Prevotella and Fusobacterium species: aprospective observational study. Anaerobe 2011, 17(3):113-117. doi:10.1016/j.anaerobe.2011.05.013.

6. Rosa RG, Goldani LZ: Cohort study of the impact of time to antibiotic administration on mortality in patients with febrile neutropenia. Antimicrob Agents Chemother 2014, 58(7):3799-803. doi:10.1128/AAC.02561-14.

7. Rosa RG, Goldani LZ, dos Santos RP: Association between adherence to an antimicrobial stewardship program and mortality among hospitalized cancer patients with febrile neutropaenia: a prospective cohort study. BMC Infect Dis 2014, 14:286. doi:10.1186/1471-2334-14-286.

8. Freifeld AG, Bow EJ, Sepkowitz KA, Boeckh MJ, Ito Jl, Mullen CA, Raad II, Rolston KV, Young JA, Wingard JR: Clinical practice guideline for the use of antimicrobial agents in neutropenic patients with cancer: 2010 update by the Infectious Diseases Society of America. Clin Infect Dis 2011, 52:e56-e93. doi:10.1093/cid/cir073.

9. De Naurois J, Novitzky-Basso I, Gill MJ, Marti FM, Cullen MH, Roila F, ESMO Gguidelines Working Group: Management of febrile neutropenia: ESMO clinical practice guidelines. Ann Oncol 2010, 21:v252-v256. doi:10.1093/ annonc/mda196.

10. Klastersky J, Paesmans M, Rubenstein EB, Boyer M, Elting L, Feld R, Gallagher J, Herrstedt J, Rapoport B, Rolston K, Talcott J: The Multinational Association for Supportive Care in Cancer Risk Index: a multinational scoring system for identifying low-risk febrile neutropenic cancer patients. J Clin Oncol 2000, 18(16):3038-3051.

11. Clinical and Laboratory Standards Institute (CLSI): Performance Standards for Antimicrobial Susceptibility Testing: Twenty-Second Informational Supplement. M100-S22. Wayne, PA: 2012

12. Garbino J, Villiger P, Caviezel A, Matulionyte R, Uckay I, Morel P, Lew D: A randomized prospective study of cefepime plus metronidazole with imipenem-cilastatin in the treatment of intra-abdominal infections. Infection 2007, 35(3):161-166.

13. Barie PS, Vogel SB, Dellinger EP, Rotstein OD, Solomkin JS, Yang JY, Baumgartner TF: A randomized, double-blind clinical trial comparing cefepime plus metronidazole with imipenem-cilastatin in the treatment of complicated intra-abdominal infections. Cefepime Intra-Abdominal Infection Study Group. Arch Surg 1997, 132(12):1294-1302.

14. Löfmark S, Edlund C, Nord CE: Metronidazole is still the drug of choice for treatment of anaerobic infections. Clin Infect Dis 2010, 50:S16-S23. doi:10.1086/647939.

doi:10.1186/1756-0500-7-614

Cite this article as: Rosa et al:: Comparison of anti-anaerobic antimicrobial strategies in cancer patients with febrile neutropenia and gastrointestinal symptoms. BMC Research Notes 2014 7:614. 